\title{
ON BAD SUPERNILPOTENT RADICALS
}

\author{
H. FRANCE-JACKSON
}

(Received 10 May 2011)

\begin{abstract}
A supernilpotent radical $\alpha$ is called bad if the class $\pi(\alpha)$ of all prime and $\alpha$-semisimple rings consists of the one-element ring 0 only. We construct infinitely many bad supernilpotent radicals which form a generalization of Ryabukhin's example of a supernilpotent nonspecial radical. We show that the family of all bad supernilpotent radicals is a sublattice of the lattice of all supernilpotent radicals and give examples of supernilpotent radicals that are not bad.
\end{abstract}

2010 Mathematics subject classification: primary 16N80.

Keywords and phrases: supernilpotent radical, special radical, prime radical, Boolean rings, prime essential rings.

\section{Introduction}

In this paper all rings are associative and all classes of rings are closed under isomorphisms and contain the one-element ring 0. A ring $A$ is called Boolean if $a^{2}=a$ for every $a \in A$. The fundamental definitions and properties of radicals can be found in [2] and [8]. A class $\mu$ of rings is called hereditary if $\mu$ is closed under ideals. If $\mu$ is a hereditary class of rings, $\mathcal{U}(\mu)$ denotes the upper radical generated by $\mu$, that is, the class of all rings which have no nonzero homomorphic images in $\mu$. For a radical $\alpha$ the class of all $\alpha$-semisimple rings is denoted by $\mathcal{S}(\alpha)$. Also, $\pi$ denotes the class of all prime rings and $\beta=\mathcal{U}(\pi)$ the prime radical. An ideal $I$ of a ring $R$ is called essential if $I \cap J \neq 0$ for any nonzero ideal $J$ of $R$. A hereditary class $\mu$ of prime rings is called special if $\mu$ is closed under essential extensions, that is, if $I \in \mu$ is an essential ideal of a ring $R$, then $R \in \mu$. The upper radical $\mathcal{U}(\mu)$ generated by a special class $\mu$ is called a special radical. A hereditary radical that contains $\beta$ is called a supernilpotent radical. We call a supernilpotent radical $\alpha$ bad if the class $\pi(\alpha)=\pi \cap \mathcal{S}(\alpha)$ consists of the one-element ring 0 only. A radical $\alpha$ that is not the class of all associative rings is called nontrivial. The $\alpha$-radical of a ring $R$ is denoted by $\alpha(R)$.

Since special radicals are hereditary and contain $\beta$, every special radical is supernilpotent. Therefore, Andrunakievich [1] asked whether every supernilpotent

(C) 2011 Australian Mathematical Publishing Association Inc. 0004-9727/2011 \$16.00 
radical is special. Examples of nonspecial supernilpotent radicals were given in $[3,4,6,7,12,14,15]$. Since a supernilpotent radical $\alpha$ is special if and only if $\alpha=\mathcal{U}(\pi(\alpha))$ [2, 8], nontrivial bad supernilpotent radicals provide the most natural counterexamples to Andrunakievich's question. The first such example was constructed by Ryabukhin [11] who showed that the upper radical generated by the class of all Boolean rings which do not contain an ideal which is a prime field with two elements is a supernilpotent but nonspecial radical. We will now give a generalization of Ryabukhin's construction which will allow us to build infinitely many nontrivial bad supernilpotent radicals. Moreover, we will show that the family of all bad supernilpotent radicals is a sublattice of the lattice of all supernilpotent radicals. Also, we will show that there exist supernilpotent radicals that are not bad.

\section{Main results}

Let $\varepsilon$ denote the class of all prime essential rings [7], that is, semiprime rings $R$ such that no nonzero ideal of $R$ is a prime ring. Let $*$ denote the class of all $*$-rings, that is, semiprime rings $R$ such that $R / I \in \beta$ for every nonzero ideal $I$ of $R$. A special class $\sigma$ of rings is called subdirectly closed if $\pi(\mathcal{U}(\sigma))=\sigma$. For example, for every natural number $n$ and any finite field $F$, the class $\left\{F_{n}\right\} \subseteq *$ consisting of the $\operatorname{ring} F_{n}$ of all $n \times n$ matrices with entries from $F$ is such a class [9].

THEOREM 1. For every nonzero subdirectly closed special class $\sigma \subseteq *$, the radical $\alpha=\mathcal{U}(\mathcal{S}(\mathcal{U}(\sigma)) \cap \varepsilon)$, is a nontrivial bad supernilpotent radical. Thus $\alpha$ is not a special radical.

Proof. To prove that $\alpha$ is nontrivial, it suffices to show that $\mathcal{S}(\mathcal{U}(\sigma)) \cap \varepsilon \neq\{0\}$. To build a nonzero ring in $\mathcal{S}(\mathcal{U}(\sigma)) \cap \varepsilon$, we will adopt the construction described in [7, Lemma 1, p. 234]. Let $R$ be a nonzero element of $\sigma$, let $\kappa$ be a cardinal number greater than the cardinality of $R$ and let $W(\kappa)$ be the set of all finite words made from a (well-ordered) alphabet of cardinality $\kappa$, lexicographically ordered. Then $W(\kappa)$ is a semigroup with multiplication defined by $x y=\max \{x, y\}$ and it follows from [7] that the semigroup ring $A=R(W(\kappa))$ is prime essential and a subdirect sum of copies of $R \in \sigma$. Thus $0 \neq A \in \mathcal{S}(\mathcal{U}(\sigma)) \cap \varepsilon$, which shows that $\alpha$ is nontrivial.

It follows from [7] that $\varepsilon$ is a weakly special class. But, since $\mathcal{U}(\sigma)$ is a special (and so supernilpotent) radical, it follows that $\mathcal{S}(\mathcal{U}(\sigma))$ is a weakly special class, too. Thus $\mathcal{S}(\mathcal{U}(\sigma)) \cap \varepsilon$ is a weakly special class. Therefore the radical $\alpha=\mathcal{U}(\mathcal{S}(\mathcal{U}(\sigma)) \cap \varepsilon)$ is a supernilpotent radical.

We will now show that $\alpha$ is bad. Suppose that it is not and let $R$ be a nonzero ring in $\pi(\alpha)$. Then $R$ is $\alpha$-semisimple and, since $\alpha$ is a supernilpotent radical determined by the weakly special class $\mathcal{S}(\mathcal{U}(\sigma)) \cap \varepsilon$, it follows that $R$ is a subdirect sum of rings $R_{\lambda} \in \mathcal{S}(\mathcal{U}(\sigma)) \cap \varepsilon \subseteq \mathcal{S}(\mathcal{U}(\sigma))$. But, being a semisimple class, $\mathcal{S}(\mathcal{U}(\sigma))$ is closed under subdirect sums. Thus $R \in \mathcal{S}(\mathcal{U}(\sigma)) \cap \pi=\sigma$. But, as $\sigma \subseteq *$, it follows that $R$ is a $*$-ring. On the other hand, since $R$ is a subdirect sum of the rings $R_{\lambda}$, there exists an ideal $I_{\lambda}$ of $R$ such that $I_{\lambda} \neq R$ and $R / I_{\lambda} \cong R_{\lambda} \in \mathcal{S}(\mathcal{U}(\sigma)) \cap \varepsilon \subseteq \mathcal{S}(\beta)$. But, since $R$ is a $*$-ring, 
we then must have $I_{\lambda}=0$ which implies that $R \in \varepsilon \cap \pi=\{0\}$, a contradiction. Thus $\pi(\alpha)=\{0\}$, which means that $\alpha$ is bad.

Since $\pi(\alpha)=\{0\}, \mathcal{U}(\pi(\alpha))$ is a trivial radical. But $\alpha$ is not, so $\alpha \neq \mathcal{U}(\pi(\alpha))$, which implies that $\alpha$ is not a special radical.

Proposition 2. For every natural number $n$ and every finite field $F$ the class $\mathcal{S}\left(\mathcal{U}\left(\left\{F_{n}\right\}\right)\right) \cap \varepsilon$ consists precisely of rings that do not contain ideals isomorphic to $F_{n}$ and are subdirect sums of copies of $F_{n}$.

Proof. Let $R \in \mathcal{S}\left(\mathcal{U}\left(\left\{F_{n}\right\}\right)\right) \cap \varepsilon$. Then $R \in \mathcal{S}\left(\mathcal{U}\left(\left\{F_{n}\right\}\right)\right)$ and so $R$ is isomorphic to a subdirect sum of copies of $F_{n}$. But we also have that $R \in \varepsilon$ so, since $F_{n} \in \pi, R$ cannot contain an ideal isomorphic to $F_{n}$. Conversely, suppose that $R$ is isomorphic to a subdirect sum of copies of $F_{n}$ and does not contain ideals isomorphic to $F_{n}$. Then $R \in \mathcal{S}\left(\mathcal{U}\left(\left\{F_{n}\right\}\right)\right)$. If $R$ contained a nonzero ideal $I \in \pi$, then $I$ would be a member of $\mathcal{S}\left(\mathcal{U}\left(\left\{F_{n}\right\}\right)\right) \cap \pi=\left\{F_{n}\right\}$ because $\mathcal{S}\left(\mathcal{U}\left(\left\{F_{n}\right\}\right)\right)$, being a semisimple class, is hereditary. This gives a contradiction. Thus $R$ is prime essential, which implies that $R \in \mathcal{S}\left(\mathcal{U}\left(\left\{F_{n}\right\}\right)\right) \cap \varepsilon$.

It is well known [10, Theorem 3.16, p. 58] that a ring $A$ is Boolean if and only if $A$ is a subdirect sum of copies of the two-element field $\mathbb{Z}_{2}$. Thus, taking $\sigma=\left\{\mathbb{Z}_{2}\right\}$ in Theorem 1, we have the following corollary.

COROllary 3 [11]. The upper radical generated by the class of all Boolean rings which do not contain an ideal which is a prime field with two elements is a supernilpotent but nonspecial radical.

It is well known [13] that the family $\mathbb{K}$ of all supernilpotent radicals is a lattice with respect to inclusion. Minimal elements of $\mathbb{K}$ are called supernilpotent atoms. Examples of supernilpotent atoms can be found in [5]. We have the following theorem.

THEOREM 4. The family $\mathbb{B}$ of all bad supernilpotent radicals is a sublattice of $\mathbb{K}$.

Proof. Let $\alpha, \gamma \in \mathbb{B}$. Then $\pi(\alpha)=\{0\}$ and $\pi(\gamma)=\{0\}$. Then, since $\mathcal{S}(\alpha \vee \gamma)=\mathcal{S}(\alpha) \cap$ $\mathcal{S}(\gamma)$, it follows that $\pi(\alpha \vee \gamma)=\{0\}$, which means that $\alpha \vee \gamma \in \mathbb{B}$.

To show that $\alpha \wedge \gamma \in \mathbb{B}$, suppose that $0 \neq R \in \pi(\alpha \wedge \gamma)$. If $\alpha(R)=0$ then, since $R \in \pi$, it follows that $R \in \pi(\alpha)$, which is impossible since $\pi(\alpha)=\{0\}$. Thus $\alpha(R)$ is a nonzero ideal of $R$. Similarly, $\gamma(R)$ is a nonzero ideal of $R$. So, since $R \in \pi$, it follows that $\alpha(R) \gamma(R)$ is a nonzero ideal of $R$. Moreover, since both $\alpha$ and $\gamma$ are hereditary radicals, it follows that $\alpha(R) \gamma(R) \in \alpha \wedge \gamma$, which contradicts the fact that $R \in \mathcal{S}(\alpha \wedge \gamma)$. Thus $\pi(\alpha \wedge \gamma)=\{0\}$, which means that $\alpha \wedge \gamma \in \mathbb{B}$.

We do not know whether $\mathbb{B}$ is a complete sublattice of $\mathbb{K}$. To answer this question in the negative, it would suffice to show that $\wedge \alpha_{p} \notin \mathbb{B}$, where $p$ is a prime, $\alpha_{p}=$ $\mathcal{U}\left(\mathcal{S}\left(\mathcal{U}\left(\left\{\mathbb{Z}_{p}\right\}\right)\right) \cap \varepsilon\right)$ and $\mathbb{Z}_{p}$ is the $p$-element field.

Our final result shows examples of supernilpotent radicals which are not bad.

THeOREM 5. If $\alpha$ is a supernilpotent atom, then $\alpha$ is not bad. 
Proof. Let $S$ be a nonzero simple prime ring. Then either $S \in \mathcal{S}(\alpha)$ or $S \in \alpha$. In the first case $S \in \pi(\alpha)$, which makes $\alpha$ not bad. In the second case we have $\beta \subsetneq \bar{l}_{A} \subseteq \alpha$, where $\bar{l}_{A}$ denotes the smallest supernilpotent radical containing $A$. So, since $\alpha$ is a supernilpotent atom, we must have $\alpha=\bar{l}_{A}$. But then every nonzero simple prime ring $R$ which is not isomorphic to $S$ is in $\pi(\alpha)$, which shows that $\alpha$ is not bad.

\section{References}

[1] V. A. Andrunakievich, 'Radicals of associative rings I', Mat. Sb. 44 (1958), 179-212 (in Russian).

[2] V. A. Andrunakievich and Yu. M. Ryabukhin, Radicals of Algebras and Structure Theory (Nauka, Moscow, 1979), (in Russian).

[3] K. I. Beidar and K. Salavova, 'Some examples of supernilpotent nonspecial radicals', Acta Math. Hungar. 40 (1982), 109-112.

[4] K. I. Beidar and R. Wiegandt, 'Radicals induced by the total of rings', Beiträge Algebra Geom. 38 (1997), 149-159.

[5] H. France-Jackson, 'On atoms of the lattice of supernilpotent radicals', Quaest. Math. 10 (1987), 251-256.

[6] H. France-Jackson, 'On prime essential rings', Bull. Aust. Math. Soc. 47 (1993), 287-290.

[7] B. J. Gardner and P. N. Stewart, 'Prime essential rings', Proc. Edinb. Math. Soc. 34 (1991), 241-250.

[8] B. J. Gardner and R. Wiegandt, Radical Theory of Rings (Marcel Dekker, New York, 2004).

[9] K. K. Krachilov, 'Coatoms of the lattice of special radicals', Mat. Issled. Kishinev 49 (1979), 80-86 (in Russian).

[10] N. H. McCoy, The Theory of Rings (Chelsea, New York, 1973).

[11] Yu. M. Ryabukhin, 'On overnilpotent and special radicals', Issled. Alg. Mat. Anal., Kishinev (1965), 65-72 (in Russian).

[12] Yu. M. Ryabukhin, 'Supernilpotent and special radicals', Mat. Issled. Kishinev 48 (1978), 80-83 (in Russian).

[13] R. L. Snider, 'Lattices of radicals', Pac. J. Math. 40 (1972), 207-220.

[14] S. Tumurbat, 'Some issues in the theory of supernilpotent radicals', Bul. Acad. Stiinte Rep. Mold. 1(38) (2002), 3-43.

[15] L. C. A. van Leeuwen and T. L Jenkins, 'A supernilpotent non-special radical class', Bull. Aust. Math. Soc. 9 (1973), 343-348.

\section{H. FRANCE-JACKSON, Department of Mathematics and Applied Mathematics, Nelson Mandela Metropolitan University, Summerstrand Campus (South), PO Box 77000, Port Elizabeth 6031, South Africa e-mail: cbf@easterncape.co.uk}

